\title{
Predictor Analysis on Non-parametric Bulk Arrival Fuzzy Queueing System
}

Sivaraman Geetha1, Bharathi Ramesh Kumar ${ }^{2 *}$ and Sankar Murugesan ${ }^{3}$

${ }^{1}$ National Engineering College, Kovilpatti, Tamil Nadu, India

${ }^{2}$ Sree Sowdambika College of Engineering, Aruppukottai, Tamil Nadu, India

${ }^{3}$ Sri.S.Ramasamy Naidu Memorial College, Sattur, Tamil Nadu, India

\begin{abstract}
In general a management does not like the arriving customer wait for service in a system. It is not possible for all the times because the situation. In this case parameter estimation is helpful to rectify this difficulty and to analyze the modeling of system performance. Practically the queue parameters are not deterministic. So in this paper we estimate the queue parameter. Initially we construct the inverse membership function of the k-phase fuzzy queueing system and proposed an algorithm of performing the system. Finally, obtained the level of uncertainty range in the system and analyze the interval optimality level of k-phase fuzzy queueing system. The idea is extended to the work. A numerical example is included.
\end{abstract}

Keywords: Fuzzy sets; Mixed integer nonlinear programming; K-phase Erlang distribution; $\alpha$ - cut Membership function

\section{Introduction}

In general, any management system did not like that the arriving customer waiting in the service stage on long time. Sometime it is not possible because of the situation due natural calamities, the server providing the worst service, service time factor, etc., In this case our system fully block to the service. So, management likes to avoid this kind of manners, in this connection we estimated the queues parameters. In this situation bulk arriving queueing model is useful for recovery the problem in this case service may talented in many phases. The Researcher [1-3] has investigated the performance level. The basic queue characters are involved the certain probability distribution. The attractiveness is analyzing the observed data through statistical interference. The observable data's unquestionable on the queueing system actually are. It is important to utilize the data of extend possible. Many algebraic problems are connected with the simulation modeling in queueing analysis. A statistical formula can support the best use of remaining data should be taken its important of the queueing studies. The initial works on the measurements of queue was totally observed a period of time and complete information was available in the form of the arrival moments and service of each customer. In general, model of queue liable on the markov process. Clarke and Benes are assumed the processing time consider as a special distribution. They are investigated the queues parameters through statistical interfering the different models $(M / M / 1)$ and $(M / M / \infty)$. In general depends on the situation queueing parameters are uncertainty. For this case fuzzy set theory is most helpful to analyze the optimality level of the system performance. In [4] classical queueing models are extended in fuzzy model with more applications. The fuzzy queuing models are more truthful for the classical ones [5-11] have analyzed and proved important results on fuzzy applications using $\alpha$-level membership function, [12-14] analyze the nonlinear programming for single phase fuzzy queues in general discipline [15] Provided the overview on the conceptual aspects for the phase service in different queueing model. Clearly, many researchers are analyzing the queueing system modeling. In this paper, we analyze the interval optimality level of k-phase fuzzy queueing system; the above work extended in $[14,16]$ and derived the uncertainty range k-phase fuzzy queueing system with the help of inverse membership function.

\section{Generalized Erlang k-phase service distribution}

In this model service time consider as an Erlang distribution. More specially, the overall rate of each service phase is $k \mu$. Even though the service may not actually contain in $\mathrm{k}$ phases, Let $p_{n, i}(t)$ be the steady state probability, here " $n, i$ " denotes customers in the system and service in k-phase. Here, we considered the number of phases in backward, so $\mathrm{k}$ is the first phase of service and one is the last phase. We can derive the steady state balance equation is:

Inter arrival time: $A(t)=\lambda e^{-\lambda t}, t \geq 0$

Service time: $B(t)=\frac{k \mu(k \mu t)^{k-1} e^{-k \mu t}}{(k-1) !} \quad t \geq 0$, here $E(x)=1 / \mu$ and $V(x)=1 / k \mu^{2}$.

The k-phase queueing system shown in Figure 1.

Define the 2-dim state variable $(n, i)$ to be the total number of customers $\mathrm{n}$ in the system and the customer being served is at $\mathrm{i}$-stage (phase). Then $P(n)=\sum_{i=1}^{k} P(n, i)$

$$
\begin{aligned}
& \left(\begin{array}{l}
i=k: \text { at the } 1 \text { st phase } \\
i=1: \text { at the last phase } \\
i=0 \text { : leaving the system or service completion }
\end{array}\right) \\
& \begin{array}{r}
p_{n, i}(t+\Delta t)=p_{n, i}(t)(1-\lambda \Delta t-k \mu \Delta t)+p_{n, i+1}(t)(k \mu \Delta t)+ \\
p_{n-1, i}(t)(\lambda \Delta t) \quad n \geq 2,1 \leq i \leq k
\end{array} \\
& \begin{array}{r}
p_{n, k}(t+\Delta t)= \\
+p_{n, k}(t)(1-\lambda \Delta t-k \mu \Delta t)+p_{n, k+1}(t)(k \mu \Delta t) \\
+p_{n-1, i}(t)(\lambda \Delta t) \quad n \geq 2,1 \leq i \leq k
\end{array}
\end{aligned}
$$

*Corresponding author: Bharathi Ramesh Kumar, Sree Sowdambika College of Engineering, Aruppukottai, Tamil Nadu, India, Tel: 984-268-9899; E-mail: brkumarmath@gmail.com

Received April 14, 2017; Accepted May 10, 2017; Published May 22, 2017

Citation: Geetha S, Kumar BR, Murugesan S (2017) Predictor Analysis on Nonparametric Bulk Arrival Fuzzy Queueing System. Global J Technol Optim 8: 212 doi: 10.4172/2229-8711.1000212

Copyright: ( 2017 Geetha S, et al. This is an open-access article distributed under the terms of the Creative Commons Attribution License, which permits unrestricted use, distribution, and reproduction in any medium, provided the original author and source are credited. 


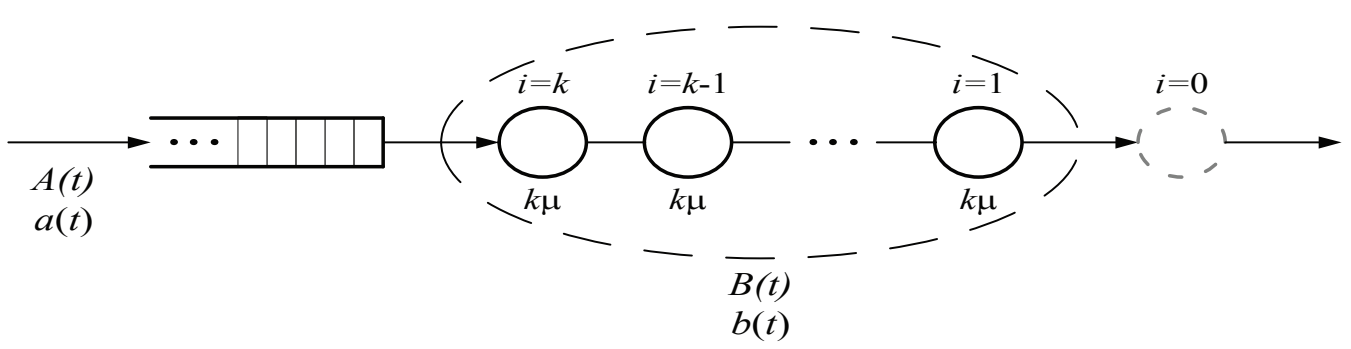

Figure 1: K-Phase queueing system.

At steady state for $\mathrm{n}>0,1 \leq i \leq k$

$-\lambda p_{0,0}+k \mu p_{1,1}=0$,

If $\mathrm{n}=1$, then

if $n \geq 2$, then

$\left\{\begin{array}{l}-(\lambda+k \mu) p_{n, i}+k \mu p_{n, i+1}+\lambda p_{n-1, i}=0 \\ -(\lambda+k \mu) p_{n, k}+k \mu p_{n+1,1}+\lambda p_{n-1, k}=0\end{array}\right.$

We obtained the total phase service is:

$$
\begin{aligned}
G(z) & =\sum_{n=1}^{\infty} \sum_{i=1}^{k} p_{n, i} z^{k(n-1)+i}+p_{0}=\frac{p_{0}(1-z)}{1-z(1+r)+r z^{k+1}} \\
W_{q}= & \sum_{n=1}^{\infty} \sum_{i=1}^{k}\left[\frac{k(n-1)+i}{k \mu}\right] p_{n, i}=\left.\frac{1}{k \mu} G^{\prime}(z)\right|_{z=1} \\
= & \mathrm{E}\left(N_{q}\right) \frac{1}{\mu}+\mathrm{E}(I) \frac{1}{k \mu}
\end{aligned}
$$

If we let $j=(n-1) k+i, n \geq 0,1 \leq i \leq k$ be the total number of phases for $(\mathrm{n}, \mathrm{i})$ state in the system with problem $p_{j}^{(p)} \cdot p_{j}=0, j>0$, $p_{n}=\sum_{i=1}^{k} p_{n, i}=\sum_{j=(n-1) k+1}^{n k} p_{j}^{(p)}$

$p_{j}^{(p)}$ : The prob of $\mathrm{j}$ in the bulk-input system. Then we rewrite

$\left\{\begin{array}{l}-\lambda p_{0}+\mu k p_{1} \quad=0 \\ -(\lambda+k \mu) p_{n}+k u p_{n+1}+\lambda p_{m-k}=0, n \geq 1\end{array}\right\}$

The Erlangian type $\mathrm{k}$ service model is equivalent to Bulk input model where $c_{k}=1, c_{x}=0, x \neq k$.

$$
p_{0}=1-\frac{\lambda \mathrm{E}(X)}{k \mu}=1-\frac{k \lambda}{k \mu}=1-\frac{\lambda}{\mu}=1-\rho
$$

Using partial fraction expansion may yield

$$
\begin{gathered}
G(z)=(1-\rho) \sum_{i=1}^{k} \frac{A_{i}}{\left(1-\frac{z}{z_{i}}\right)} \quad \text { where } \quad A_{i}=\prod_{\substack{n=1 \\
n \neq i}}^{k} \frac{1}{\left(1-\frac{z_{i}}{z_{n}}\right)} \text { and } \\
p_{j}^{(P)}=(1-\rho) \sum_{i=1}^{k} A_{i}\left(z_{i}\right)^{-j} ; \quad p_{n}=\sum_{j=(n-1) k+1}^{n-k} P_{j}^{(P)}
\end{gathered}
$$

The performance measures as follows:

$$
\begin{aligned}
W_{q} & =\mathrm{E}\left(T_{q}\right)=\mathrm{E}\left(N_{q}\right) \frac{1}{\mu}+\mathrm{E}(I) \frac{1}{k \mu} \\
& =\sum_{n=1}^{\infty} \sum_{i=1}^{k}\left[\frac{k(n-1)+i}{k \mu}\right] p_{n, i}+0 \cdot p_{0} \\
& =\left.\frac{1}{k \mu} \mathrm{G}^{\prime}(\mathrm{z})\right|_{z=1}
\end{aligned}
$$

$$
\begin{aligned}
& \left.G^{\prime}(z)\right|_{z=1}=\frac{(k+1) \rho}{2(1-\rho)}, W_{q}=\frac{(k+1) \rho}{2 k \mu(1-\rho)}=\frac{k+1}{2 k} \cdot \frac{\lambda}{\mu(\mu-\lambda)} \\
& L_{q}=\lambda W_{q}=\frac{k+1}{2 k} \cdot \frac{\lambda^{2}}{\mu(\mu-\lambda)} \\
& W=W_{q}+\frac{1}{\mu}=\frac{k+1}{2 k} \cdot \frac{\lambda}{\mu(\mu-\lambda)}+\frac{1}{\mu} \\
& L=\lambda W=\frac{k+1}{2 k} \cdot \frac{\lambda^{2}}{\mu(\mu-\lambda)}+\frac{\lambda}{\mu} \\
& G^{\prime}(z)=\frac{(k+1) \lambda}{2(\mu-\lambda)} \text { - Denotes the average total phase in the system. }
\end{aligned}
$$

\section{Fuzzy queues with k-phase infinite capacity}

Consider the arrival and service rates of $\mathrm{k}$-phase queueing systems in triangular fuzzy number and defined as $\bar{\lambda}=\left\{\left(x, \mu_{\bar{\lambda}}(x) / x \in X\right\}\right.$ , $\bar{\mu}=\left\{\left(x, \mu_{\bar{\mu}}(y) / y \in Y\right\}\right.$ here $\mu_{\bar{\lambda}}(x)$ and $\mu_{\bar{\mu}}(y)$ being the membership function of the arrival and service rate. Let $\mathrm{P}$ denote measuring the queueing system performance and defined the fuzzy system is

$$
\mu_{p(\bar{\lambda}, \bar{\mu})}(z)=\sup \min \left\{\mu_{\bar{\lambda}}(x), \mu_{\mu}(y) / z=p(x, y)\right\}
$$

Equation $[9,10]$ formulated the fuzzy membership function as follows:

$$
\begin{aligned}
& \mu_{w_{q}}(z)=\sup \min \left\{\mu_{\bar{\lambda}}(x), \mu_{\mu}(y) / z=\left(\frac{k+1}{2 k}\right) \frac{\rho}{\mu(1-\rho)}\right\} \\
& \mu_{\overline{L_{q}}}(z)=\sup \min \left\{\mu_{\bar{\lambda}}(x), \mu_{\mu^{-}}(y) / z=\lambda \overline{w_{q}}\right\}
\end{aligned}
$$

Now, the idea is establish the mathematical programming technique a pair of nonlinear programs is developed and the different possibility levels are calculated. So, we estimated the system performance through the statistical interference.

\section{Solution Procedure}

To derive the inverse membership function of $P(\bar{\lambda}, \bar{\mu})$ on the basis of $\alpha$-cuts. The fuzzy arrival and service are defined as follows:

$$
\begin{aligned}
& \overline{\lambda_{\alpha}}=\left[x_{\alpha}^{l}, x_{\alpha}^{u}\right]=\left[\min \left\{x / \mu_{\lambda}(x) \geq \alpha\right\}, \max \left\{x / \mu_{\lambda}(x) \geq \alpha\right\}\right] \\
& \overline{\mu_{\alpha}}=\left[y_{\alpha}^{l}, y_{\alpha}^{u}\right]=\left[\min \left\{x / \mu_{\mu}(y) \geq \alpha\right\}, \max \left\{y / \mu_{\mu}(y) \geq \alpha\right\}\right]
\end{aligned}
$$

In [16], represented the queue parameter in different possibility levels of intervals, therefore; FM/FEk/1 can be summarized the family of crisp M/Ek/1 queues in the a-level sets. The Appearance of above two sets is relation between ordinary and fuzzy sets [16]. The bounds of $\alpha$ cut fuzzy interval obtained as $x_{\alpha}^{l}=\min \left(\mu_{\lambda}^{-1}(\alpha), x_{\alpha}^{u}=\max \left(\mu_{\lambda}^{-1}(\alpha)\right.\right.$, $y_{\alpha}^{l}=\min \left(\mu_{\mu}^{-1}(\alpha)\right.$ and $y_{\alpha}^{u}=\max \left(\mu_{\mu}^{-1}(\alpha)\right.$. Clearly, defined the membership function of $(\bar{\lambda}, \bar{\mu})$ is also parameterized by $\alpha$. Therefore, rendering to (i), $\mu_{\bar{L}_{q}}(z)$ and $\mu_{w_{q}}(z)$ is the minimum of $\mu_{\bar{\lambda}}(x)$ and $\mu_{\bar{\mu}}(y)$. We need to either $\mu_{\bar{\lambda}}(x)=\alpha$ and $\mu_{\bar{\mu}}(y) \geq \alpha$ or $\mu_{\bar{\lambda}}(x) \geq \alpha$ and $\mu_{\bar{\mu}}(y)=\alpha$ such that $L_{q}(z)$ 
Citation: Geetha S, Kumar BR, Murugesan S (2017) Predictor Analysis on Non-parametric Bulk Arrival Fuzzy Queueing System. Global J Technol Optim 8: 212. doi: 10.4172/2229-8711.1000212

Page 3 of 5

and $W_{q}(z)$ to satisfy that $\mu_{L_{q}}(z)=\alpha$ and $\mu_{W_{q}}(z)=\alpha$. From (i), $y \in \mu(\alpha)$ and $x \in \lambda(\alpha)$ improved by $x \in\left[x_{\alpha}^{l}, x_{\alpha}^{u}\right]$ and $y \in\left[y_{\alpha}^{l}, y_{\alpha}^{u}\right]$ respectively, so, $\mu_{L_{q}}(z)$ and $\mu_{W_{q}}(z)$ can be written as

$$
\begin{aligned}
& L_{q_{\bar{\alpha}}}^{l}(z)=\min \left\{\left(\frac{k+1}{2 k}\right) \frac{\rho}{\mu(1-\rho)}\right\} \\
& L_{q_{\bar{\alpha}}}^{u}(z)=\max \left\{\left(\frac{k+1}{2 k}\right) \frac{\rho}{\mu(1-\rho)}\right\}
\end{aligned}
$$

Many researchers are proposed that the different methods for solving these problems. If $L_{q}(z)$ and $W_{q}(z)$ are invertible with respect to $\alpha$, then the shape of the function is

$[\mathrm{L}(\mathrm{z}), \mathrm{R}(\mathrm{z})]=L_{q_{\alpha}}^{-1} \quad$ and $\quad[\mathrm{L}(\mathrm{z}), \mathrm{R}(\mathrm{z})]=W^{-1} q_{\alpha} \quad$ obtained from membership function $\mu_{W_{q}}(z)$ and $\mu_{L_{q}}(z)$ constructed:

$$
\mu_{\mathrm{L}_{\mathrm{q}}}(z) \& \mu_{\mathrm{W}_{\mathrm{q}}}(z)=\left\{\begin{array}{ll}
L(z) & z_{1} \leq z \leq z_{2} \\
1 & z=z_{2} \\
R(z) & z_{2} \leq z \leq z_{3}
\end{array}\right\}
$$

Otherwise, the values of $L_{q_{\alpha}}$ and $W_{q_{\alpha}}$ cannot obtained analytically, the numerical solutions for $L_{q_{\alpha}}=\left[L_{q_{\alpha}}{ }^{l}, L_{q_{\alpha}}{ }^{u}\right]$ and $W_{q_{\alpha}}=\left\lfloor w_{q_{\alpha}}^{l}, w_{q_{\alpha}}{ }^{u}\right\rfloor$ at different possibility level of $a$ can be collected to approximate the shapes of $\mathrm{L}(\mathrm{z})$ and $\mathrm{R}(\mathrm{z})$. The fuzziness values are converted to crisp value using Robust Ranking Technique and we estimated the queues parameters used in statistical interference.

\section{Numerical Example}

Consider the centralize three parallel processing system in which the arrival at different level of phases. The arrival and service rate are triangular fuzzy number and the service distribution follows an Erlang distribution. The rates of arrival and service $\lambda=[1,5,7] \& \mu=[9,11]$ per minute respectively, the system manager wants to evaluate the performance measures of the system such as the expected number of customers in the queue and waiting in the queue and to analyze optimality level of the system. It is clear the system consisting three phases and the steady state condition is $\rho=\frac{3 x}{y}<1$, our proposed methodology $\overline{L_{q}}$ can be formulated as follows:

$$
{ }^{L} L_{q}(\alpha)=\frac{32 \alpha^{2}+16 \alpha+2}{15 \alpha^{2}-195 \alpha+330} \&{ }^{U} L_{q}(\alpha)=\frac{8 \alpha^{2}-56 \alpha+98}{9 \alpha^{2}+87 \alpha+54}
$$

The inverse functions of ${ }^{U} L_{q}(\alpha)$ and ${ }^{L} L_{q}(\alpha)$ exist which give the membership function $\mu_{\bar{L}_{q}}(Z)$ as

$$
\mu_{L_{q}}(Z)=\left\{\begin{array}{cc}
\frac{(195 z+16) \pm \sqrt{18225 z^{2}+48600 z}}{30 z-64} & \text { for } \frac{1}{165} \leq z \leq \frac{1}{3} \\
1 \quad \text { for } \frac{1}{3} \leq z \leq \frac{49}{27} \\
\frac{-(87 z+56) \pm \sqrt{5625 z^{2}+15000 z}}{18 z-16} & \text { for } \frac{49}{27} \leq z \leq 3
\end{array}\right.
$$

Similarly we derived,

$$
{ }^{L} w_{q}(\alpha)=\frac{8 \alpha+2}{15 \alpha^{2}-195 \alpha+330}{ }^{\&}{ }_{U} w_{q}(\alpha)=\frac{14-4 \alpha}{9 \alpha^{2}+87 \alpha+54}
$$

The Inverse function of is ${ }^{L} w_{q}(\alpha)$ and ${ }^{U} w_{q}(\alpha)$ exist, which give the membership function

$$
\mu_{\overline{\bar{q}_{q}}}(Z)=\left\{\begin{array}{cc}
\frac{(195 z+8) \pm \sqrt{18225 z^{2}+3240 z+64}}{30 z} & \text { for } \frac{1}{165} \leq z \leq \frac{13}{150} \\
1 & \text { for } \frac{13}{150} \leq z \leq \frac{1}{15} \\
\frac{-(87 z+4) \pm \sqrt{5625 z^{2}+1200 z+16}}{18 z} & \text { for } \frac{1}{15} \leq z \leq \frac{7}{27}
\end{array}\right.
$$

We analyze system performance optimality (XL-Stat 2016) relation between the average number of customer waiting in the queue and customer has to spend the time in queue shown in Figure 2 and Table 3. The classification system performance in tree structure

\section{Conclusion}

In this study, we considered an optimal K-Policy in the FM/FE(k)/1 queue with general server setup time. Especially, the entire queueing system performances are based in the server providing the service status. The Interval Optimization status if $\alpha=0$ then $\overline{L_{q}} \& \overline{W_{q}}$ is $[0.0061$ $1.8148]$ and $[0.0061,0.2593]$ respectively as shown in Table 1 . If, the degree of certainty $\alpha=1$ then $\overline{L_{q}} \& \overline{W_{q}}$ is $0.3333 \& 0.0667$ respectively. The fuzziness values are converted to crisp value using robust ranking function the value are $\overline{W_{q}} \& \overline{L_{q}}$ is $0.1327 \& 0.91945$ shown in Table 2. So,

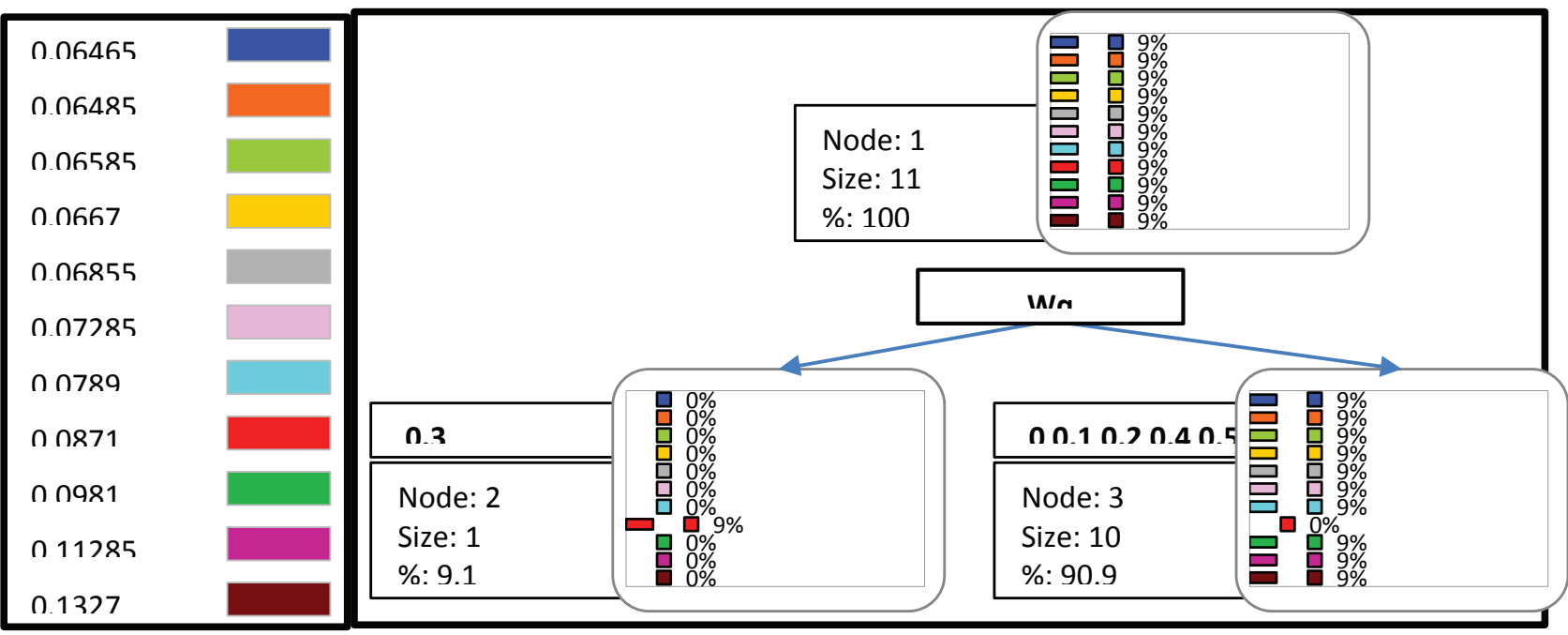

Figure 2: Performance classification of tree structure. 
Citation: Geetha S, Kumar BR, Murugesan S (2017) Predictor Analysis on Non-parametric Bulk Arrival Fuzzy Queueing System. Global J Technol Optim 8: 212. doi: 10.4172/2229-8711.1000212

Page 4 of 5

\begin{tabular}{|c|c|c|c|c|c|c|c|c|}
\hline$\alpha$ & $l_{x}^{(\alpha)}$ & $u_{x}^{(\alpha)}$ & $l_{y}^{(\alpha)}$ & $u_{y}^{(\alpha)}$ & ${ }^{L} L_{q}(\alpha)$ & ${ }^{U} L_{q}(\alpha)$ & ${ }^{L} w_{q}(\alpha)$ & ${ }^{U} w_{q}(\alpha)$ \\
\hline 0 & 1 & 7 & 9 & 11 & 0.0061 & 1.8148 & 0.0061 \\
\hline 0.1 & 1.4 & 6.8 & 9.1 & 10.9 & 0.0126 & 1.4728 & 0.0091 \\
\hline 0.2 & 1.8 & 6.6 & 9.2 & 10.8 & 0.0222 & 1.2140 & 0.0123 \\
\hline 0.3 & 2.2 & 6.4 & 9.3 & 10.7 & 0.0355 & 1.0125 & 0.0161 \\
\hline 0.4 & 2.6 & 6.2 & 9.4 & 10.6 & 0.0531 & 0.8520 & 0.0204 & 0.1839 \\
\hline 0.5 & 3 & 6 & 9.5 & 10.5 & 0.0762 & 0.7218 & 0.0254 \\
\hline 0.6 & 3.4 & 5.8 & 9.6 & 10.4 & 0.1059 & 0.6148 & 0.0311 \\
\hline 0.7 & 3.8 & 5.6 & 9.7 & 10.3 & 0.1438 & 0.5254 & 0.0378 \\
\hline 0.8 & 4.2 & 5.4 & 9.8 & 10.2 & 0.1922 & 0.4508 & 0.1203 \\
\hline 0.9 & 4.6 & 5.2 & 9.9 & 10.1 & 0.2539 & 0.3874 & 0.0458 \\
\hline 1 & 5 & 5 & 10 & 10 & 0.3333 & 0.3333 & 0.0552 \\
\hline
\end{tabular}

Table 1: The $\alpha$ cuts of the performance measures $L_{q}^{\alpha} \& W_{q}^{\alpha}$.

\begin{tabular}{|c|c|c|c|c|}
\hline$\alpha$ & $R\left(L_{q}^{\alpha}\right)$ & $R\left(W_{q}^{\alpha}\right)$ & $\alpha$ & $R\left(L_{q}^{\alpha}\right)$ \\
\hline 0 & 0.1327 & 0.91945 & 0.6 & 0.06855 \\
\hline 0.1 & 0.11285 & 0.7427 & 0.7 & 0.06585 \\
\hline 0.2 & 0.0981 & 0.6181 & 0.8 & 0.06465 \\
\hline 0.3 & 0.0871 & 0.524 & 0.9 & 0.06485 \\
\hline 0.4 & 0.0789 & 0.45255 & 1 & 0.3215 \\
\hline
\end{tabular}

Table 2: Uncertainty performance measures.

\begin{tabular}{|c|c|c|c|c|}
\hline Node & Object & Percentage & Split Variable & Values \\
\hline 1 & 11 & 100 & Lq & Wurity\% \\
\hline 2 & 1 & 9.09 & Wq & 0.3 \\
\hline 3 & 10 & 90.01 & Wq & $0.1,0.2 \& 0.4$ to 10 \\
\hline
\end{tabular}

Table 3: Purity level of interval [Lq \& Wq].

\begin{tabular}{|c|c|c|c|c|c|c|c|c|c|c|c|c|}
\hline & 0.064 & 0.064 & 0.065 & 0.06 & 0.068 & 0.072 & 0.078 & 0.087 & 0.098 & 0.112 & 0.13 & $\%$ Correct \\
\hline 0.064 & 1 & 0 & 0 & 0 & 0 & 0 & 0 & 0 & 0 & 0 & 0 & 100 \\
\hline 0.064 & 1 & 0 & 0 & 0 & 0 & 0 & 0 & 0 & 0 & 0 & 0 & 0 \\
\hline 0.065 & 1 & 0 & 0 & 0 & 0 & 0 & 0 & 0 & 0 & 0 & 0 & 0 \\
\hline 0.066 & 1 & 0 & 0 & 0 & 0 & 0 & 0 & 0 & 0 & 0 & 0 & 0 \\
\hline 0.068 & 1 & 0 & 0 & 0 & 0 & 0 & 0 & 0 & 0 & 0 & 0 & 0 \\
\hline 0.072 & 1 & 0 & 0 & 0 & 0 & 0 & 0 & 0 & 0 & 0 & 0 & 0 \\
\hline 0.078 & 1 & 0 & 0 & 0 & 0 & 0 & 0 & 0 & 0 & 0 & 0 & 0 \\
\hline 0.087 & 0 & 0 & 0 & 0 & 0 & 0 & 0 & 1 & 0 & 0 & 0 & 100 \\
\hline 0.098 & 1 & 0 & 0 & 0 & 0 & 0 & 0 & 0 & 0 & 0 & 0 & 0 \\
\hline 0.112 & 1 & 0 & 0 & 0 & 0 & 0 & 0 & 0 & 0 & 0 & 0 & 0 \\
\hline 0.132 & 1 & 0 & 0 & 0 & 0 & 0 & 0 & 0 & 0 & 0 & 0 & 0 \\
\hline Total & 10 & 0 & 0 & 0 & 0 & 0 & 0 & 1 & 0 & 0 & 0 & 18.18 \\
\hline
\end{tabular}

Table 4: Interval optimal value.

performance of the system is not optimized, because the frequency of the system is 0.954 and the estimation of the sample percentage level $W_{q}$ is not good because level is reached only $18.18 \%$ (based on the estimation Table 4 degree of certainty not good). Our proposed methodology is most helpful for the system operation (simulation) studies and then we quickly identified blocking to the system.

\section{References}

1. Ke JC (2004) Bi-level control for batch arrival queues with an early setup an un-reliable server. Appl Mat Model 28: 469-485.

2. Li QL (1995) Queueing system PH/PH (PH/PH)/1 with the repairable server. Mathematical Statistics and Applied Probability 10: 75-83.

3. Hur S, Paik SJ (1999) The effect of different arrival rate on the N-policy of $M / G / 1$ with server setup. Appl Mat Model 23: 255-343.
4. Zadeh LA (1978) Fuzzy sets as a basis for a theory of possibility. Fuzzy Sets Syst 01: 3-28.

5. Buckley JJ (1990) Elementary queueing theory based on possibility theory Fuzzy Set Syst 37: 43-52.

6. Chanas S, Nowakowski M (1988) Single value simulation on fuzzy variable. Fuzzy Set Syst 21: 43-57.

7. Li RJ, Lee ES (1989) Analysis of fuzzy queues. Comp Mat App 17: 1143-1147.

8. Negi DS, Lee E (1992) Analysis and simulation of fuzzy queues. Fuzzy Sets Syst 46: 321-330.

9. Prade HM (1980) An outline of Fuzzy or possibilistic model for queueing systems, in: Wang.P.P, Chang.S.K(Eds.). Fuzzy Sets, Plenum Press, New York, 147-153.

10. Sahaya SA, Anitha N (2015) Solving a interval linear programming problem using alpha-cut operation. Int J Comp App 112: 14-16. 
Citation: Geetha S, Kumar BR, Murugesan S (2017) Predictor Analysis on Non-parametric Bulk Arrival Fuzzy Queueing System. Global J Technol Optim 8: 212. doi: 10.4172/2229-8711.1000212

Page 5 of 5

11. Stanford RE (1982) The set of limiting distributions for a Markov chain with fuzzy transition probabilities. Fuzzy Sets Syst 7: 71-78.

12. Jo JB, Tsujimura Y, Gen M, Yamazaki G (1996) Performance evaluation of networks based on Fuzzy Queueing System. Jpn J Fuzzy Theor Syst 8: 393408

13. Kao C, Li CC, Chen SP (1999) Parametric programming to the analysis of fuzzy queues. Fuzzy Sets Syst 107: 93-100.
14. Chen SP (2014) Parametric nonlinear programming for analyzing fuzzy queues with finite capacity. European Journal of Operation Research 157: 429-438.

5. Sharma R (2014) Mathematical analysis of queue with phase service. Advances in Operations Research 1-19.

16. Chen SP (2006) A bulk arrival queueing model with fuzzy parameters and varying batch sizes. Appl Mat Model 30: 920-929. 